\title{
Switchable Scattering Meta-Surfaces for Broadband Terahertz Modulation
}

SUBJECT AREAS:

OPTICS AND PHOTONICS

MATERIALS SCIENCE

Received

14 April 2014

Accepted

27 June 2014

Published

16 July 2014

Correspondence and requests for materials should be addressed to M.J. (mjarrahi@umich.

edu)

* These authors contributed equally to this work.

\author{
M. Unlu*, M. R. Hashemi*, C. W. Berry, S. Li, S.-H. Yang \& M. Jarrahi
}

Electrical Engineering and Computer Science Department, University of Michigan, Ann Arbor, Ml 48109, United States.

Active tuning and switching of electromagnetic properties of materials is of great importance for controlling their interaction with electromagnetic waves. In spite of their great promise, previously demonstrated reconfigurable metamaterials are limited in their operation bandwidth due to their resonant nature. Here, we demonstrate a new class of meta-surfaces that exhibit electrically-induced switching in their scattering parameters at room temperature and over a broad range of frequencies. Structural configuration of the subwavelength meta-molecules determines their electromagnetic response to an incident electromagnetic radiation. By reconfiguration of the meta-molecule structure, the strength of the induced electric field and magnetic field in the opposite direction to the incident fields are varied and the scattering parameters of the meta-surface are altered, consequently. We demonstrate a custom-designed meta-surface with switchable scattering parameters at a broad range of terahertz frequencies, enabling terahertz intensity modulation with record high modulation depths and modulation bandwidths through a fully integrated, voltage-controlled device platform at room temperature.

econfigurable metamaterials, artificial electromagnetic media with tunable and switchable electromagnetic characteristics, have attracted extensive attention due to their unique capabilities for routing and manipulating electromagnetic waves. Tuning the electromagnetic characteristics of metamaterials can be achieved by controlling the structure and arrangement of their so called meta-molecule building blocks. In this regard, a number of tunable metamaterials based on phase-change materials $s^{1-4}$, semiconductors ${ }^{5-7}$, graphene $e^{8-10}$, superconductors ${ }^{11-13}$, and electromechanical structures ${ }^{14-20}$ activated by optical, electrical, magnetic, and thermal stimuli have been demonstrated. Consequently, new paradigms for switching electromagnetic waves and tuning electromagnetic phase, polarization, propagation direction, and beam shape have emerged, which would not have been possible by use of naturally existing substances ${ }^{21}$. In spite of the significant progress in the field, the demonstrated tunable metamaterials are often limited in their operation bandwidth, mainly due to the resonant nature of their meta-molecule constituents. To attain the flexible design space that transformation optics demands, new artificial materials with extraordinary tunability in scattering properties over a broad range of frequencies are required.

Extreme switching of the scattering parameters over a broad range of frequencies is a property that has been only offered by superconductors when cooled below their critical temperatures ${ }^{22}$, phase-transition metal-oxides under thermal, electrical, and optical stimuli ${ }^{23-26}$, and semiconductors under optical stimuli. Here we demonstrate broadband switching of the scattering parameters at room temperature, through a new class of electricallyreconfigurable meta-surfaces. By reshaping the structural configuration of the strongly coupled meta-molecule unit cells, their collective response to an incident electromagnetic wave is altered and the surface impedance of the meta-surface is varied between a capacitive state and an inductive state within the device operation frequency. Accordingly, efficient electromagnetic flux transmission through the meta-surface and strong electromagnetic flux reflection from the meta-surface region is observed in the capacitive and inductive states, respectively.

Dynamic switching of the meta-surface scattering parameters over a broad range of frequencies will offer new opportunities for signal handling, electromagnetic switching, and beam shaping. We demonstrate a fully integrated and voltage-controlled platform for modulating the intensity of terahertz waves interacting with a customdesigned meta-surface with switchable scattering characteristics, enabling terahertz intensity modulation with record high modulation depths $(>70 \%)$ and modulation bandwidths $(>1.5 \mathrm{THz})$ at room temperature.

\section{Results}

The schematic diagram and operation principle of the meta-surface with switchable scattering parameters is shown in Fig. 1a. It consists of an array of vertically-oriented (along the $y$-axis) Au membranes, suspended above a high-resistivity Si substrate. The anchors of the suspended membranes are placed on a $\mathrm{SiO}_{2}$ layer to electrically 


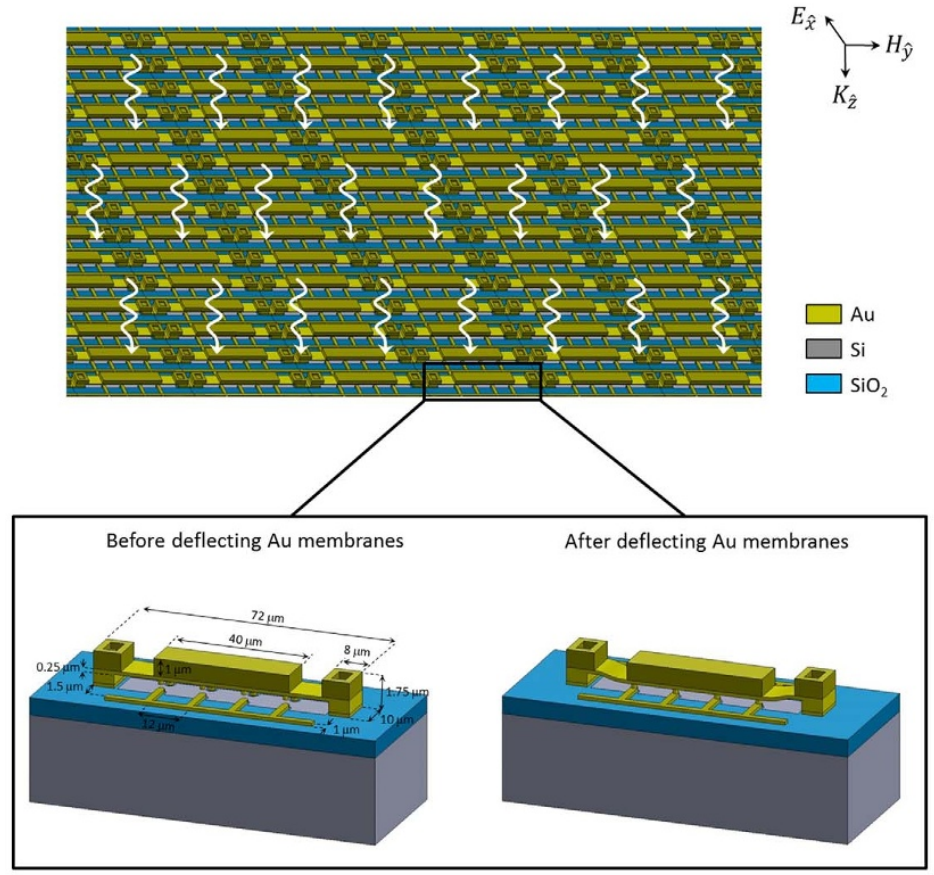

b

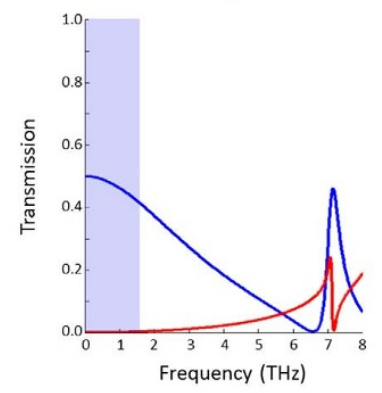

d

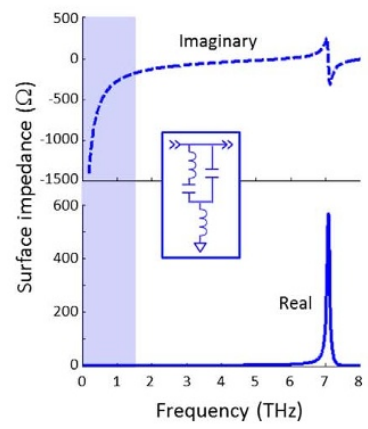

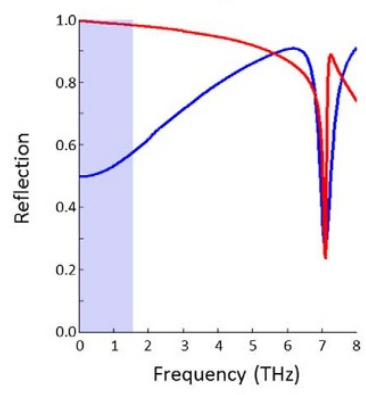

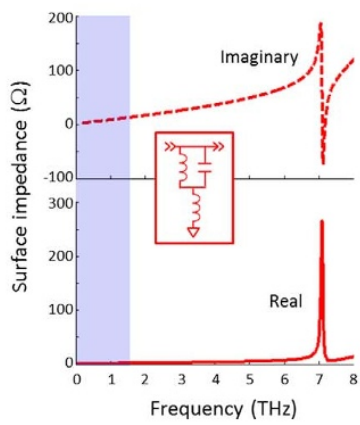

Figure 1 The operation principle of the meta-surface with switchable scattering parameters. (a) The schematic of the meta-surface, which consists of an array of vertically-oriented Au membranes suspended above a Si substrate. Depending on the voltage difference between the Au membranes and the Si substrate, the Au membranes can be suspended above the Si substrate or be in contact with an array of horizontally-oriented Au patches on the substrate. A relatively thick metal layer is used in the center of the Au membranes to assist with the flatness of the contact areas and achieve high spring constants required for high speed switching. The gap between the Au membranes and the substrate $(0.5 \mu \mathrm{m})$, and the contact dimple height $(0.25 \mu \mathrm{m})$ are chosen to achieve low switching voltages $(30 \mathrm{~V})$ and high switching speeds $(>20 \mathrm{KHz})$ while accounting for possible bending in the Au moving membranes as a result of uncompensated stress of the Au membranes. The calculated transmission and reflection scattering parameters of the designed meta-surface for a horizontally-polarized incident electromagnetic wave before deflection of the Au membranes (blue curves) and after deflection of the Au membranes (red curves) are illustrated in (b) and (c), respectively. The extracted surface impedance of the designed meta-surface before deflection of the Au membranes (blue curves) and after deflection of the Au membranes (red curves) are illustrated in (d) and (e), respectively. Electromagnetic wave interaction with the designed reconfigurable meta-surface can be also explained by the meta-surface equivalent circuit model (inset), where the horizontally-oriented metal lines function as inductors and the horizontal gaps between metal stripes function as capacitors. In the $0.1-1.5 \mathrm{THz}$ frequency range, the meta-surface equivalent circuit model is capacitive before deflection of the Au membranes and inductive after deflection of the Au membranes (Supplementary Fig. S2).

isolate them from the underlying Si substrate. When a voltage difference is applied between the Au membranes and the Si substrate, the induced electrostatic force deflects the Au membranes, moving them into contact with an array of horizontally-oriented (along the $x$-axis) $\mathrm{Au}$ patches on the $\mathrm{SiO}_{2}$ layer ${ }^{27}$. All the $\mathrm{Au}$ membranes and patches are connected through vertically-oriented Au traces such that the entire array of suspended membranes can be deflected simultaneously by controlling the voltage difference between the metasurface and the underlying Si substrate. Moreover, since the device does not draw any current before and after deflection of the $\mathrm{Au}$ membranes, a uniform voltage difference can be applied between the Au membranes and the high-resistivity Si substrate.

When an electromagnetic wave impinges on the top surface of the meta-surface, a surface electric current is induced on the metal surface, generating electric and magnetic fields on the top and bottom sides of the meta-surface. The magnitude and direction of the induced electric and magnetic fields determine the scattering properties of the structure. Before deflecting the Au membranes, the strength of the induced electric and magnetic fields on the top and bottom sides of the meta-surface in response to a horizontally-polarized incident electromagnetic wave is weak for subwavelength metamolecule feature sizes in the horizontal direction. This is the result of the metal discontinuities in the horizontal direction, which prevent the metal electrons from forming a surface electric current. In this state, an efficient electromagnetic flux transmission through the meta-surface is achieved over a frequency band inversely proportional to the meta-molecule feature size in the horizontal direction ${ }^{28}$. The reconfigurable meta-surface is designed to have deep subwavelength meta-molecule feature sizes in the horizontal direction within the device operation frequency range. Therefore, the electromagnetic interaction with the meta-surface in this state is dominated by electric dipoles induced between metal discontinuities and the surface impedance of the meta-surface is capacitive within the device operation frequency range (Supplementary Fig. S1). After deflecting the $\mathrm{Au}$ membranes and allowing them to contact the $\mathrm{Au}$ patches, the metal discontinuities in the horizontal direction are eliminated, allowing the metal electrons to form a surface electric current that produces considerable electric and magnetic fields on the top and bottom side of the meta-surface. In this state, the strength of the induced electric and magnetic fields in response to the horizontally-polarized incident electromagnetic wave is strong for subwavelength meta-molecule feature sizes in the vertical direction. This is the result of efficient energy transfer from the incident electromagnetic wave to the surface electric current, producing opposing electric and magnetic fields that cancel the incident fields on the bottom side of the meta-surface. In this state, an efficient electromagnetic flux reflection from the top of the meta-surface is achieved over a frequency band inversely proportional to the meta-molecule feature 

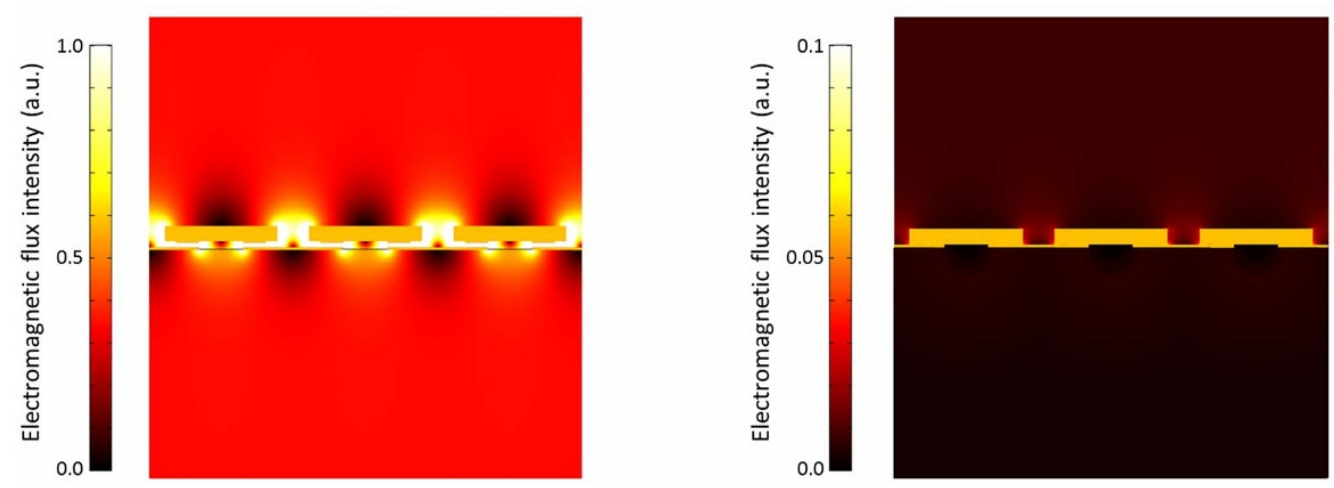

Figure $2 \mid$ Cross-sectional view of electromagnetic interaction with the presented meta-surface with switchable scattering parameters. The interaction is shown at a frequency within the operation bandwidth of the reconfigurable meta-surface $(0.2 \mathrm{THz})$, where the surface impedance of the meta-surface is varied between a capacitive state and an inductive state before and after deflection of the Au membranes, respectively. (a) Electromagnetic flux penetrates through the meta-surface before deflecting the Au membranes (capacitive state). (b) Electromagnetic flux is forced to reflect back from the meta-surface region after deflecting the Au membranes (inductive state).

size in the vertical direction ${ }^{28}$. The reconfigurable meta-surface is designed to have deep subwavelength meta-molecule feature sizes in the vertical direction within the device operation frequency range. Therefore, the electromagnetic interaction with the meta-surface in this state is dominated by the induced surface electric current and the surface impedance of the meta-surface is inductive within the device operation frequency range (Supplementary Fig. S1).

A unique advantage of the presented reconfigurable meta-surface is that the switching of the scattering parameters is exhibited over a frequency band set by the meta-surface feature size in the vertical and horizontal direction. Therefore, it can offer broadband switching through structural miniaturization. By appropriate choice of the vertical distance between the Au patches $(12 \mu \mathrm{m})$ and the horizontal distance between the Au membranes $(16 \mu \mathrm{m})$, we have designed a reconfigurable meta-surface with broadband switchable scattering parameters over a $1.5 \mathrm{THz}$ frequency band. Using a finite-element-based full-wave electromagnetic solver (ANSYS HFSS), we have analyzed the interaction of a horizontally-polarized electromagnetic wave with the designed meta-surface and extracted its surface impedance from the calculated scattering parameters. Figures $1 \mathrm{~b}$ and 1c show the estimated electromagnetic power transmission through the designed reconfigurable meta-surface and electromagnetic power reflection from the designed reconfigurable meta-surface for a horizontally-polarized incident electromagnetic wave, respectively. It shows the capability of the designed reconfigurable meta-surface for modulating the intensity of terahertz waves with more than $90 \%$ modulation depth, over a $1.5 \mathrm{THz}$ frequency band. This unprecedented modulation performance is made possible by the broadband switching of the scattering parameters of the designed reconfigurable meta-surface and can be extended even further to offer higher modulation depths and modulation bandwidths through further structural miniaturization. The 50\% electromagnetic flux transmission before deflection of the Au membranes is bound by the Fresnel reflections at the Si-air interfaces of the designed reconfigurable meta-surface mounted on a Si lens. The $3 \mathrm{~dB}$ signal loss associated with Fresnel reflections at $\mathrm{Si}$-air interfaces can be minimized by thinning the $\mathrm{Si}$ substrate under the device active area.

The extracted surface impedance of the designed meta-surface indicates extreme switching from a capacitive state (Fig. 1d) to an inductive state (Fig. 1e) over the device operation frequency band of 0.1-1.5 THz (Supplementary Fig. S2). As a result, an efficient elec- tromagnetic flux transmission through the meta-surface and a strong electromagnetic flux reflection from the meta-surface region are exhibited in the capacitive and inductive states, respectively (Fig. 2).

It should be mentioned that switching of the scattering parameters of the designed meta-surface is achieved not only through complete physical contact between the Au membranes and Au patches, but also exhibited for nanometer-scale metal separations. For nanometer-scale separations, the large capacitance between the $\mathrm{Au}$ membranes and Au patches shunts the two metal layers at high frequencies (Supplementary Fig. S3). This allows switching of the scattering parameters at relatively low voltages and enables reliable device operation by eliminating the need for applying high electrostatic forces and direct metal-to-metal contact ${ }^{29}$.

The extraordinary switching of the scattering parameters of the presented meta-surface has been utilized to develop a room-temperature terahertz intensity modulator with unprecedented modulation depth and bandwidth. The modulator prototype is fabricated on a high-resistivity Si substrate. The fabrication process starts with defining the $\mathrm{SiO}_{2}$ isolation layer areas by $\mathrm{Si}$ reactive ion etching (Fig. 3a), followed by $\mathrm{SiO}_{2}$ deposition, using plasma enhanced chemical vapor deposition. Subsequently, the wafer is planarized, using chemical mechanical polishing (Fig. 3b). The horizontally-oriented Au patches and bias lines are then formed by sputtering $\mathrm{Ti} / \mathrm{Au} / \mathrm{Ti}$ (100/1000/100 A) followed by lift-off (Fig. 3c). A PMMA/PMGI sacrificial layer $(0.5 \mu \mathrm{m})$ is then spin coated and patterned for the anchor areas (Fig. 3d) and contact dimples (Fig. 3e) using two separate masks. Next, a Ti/Au layer $(100 / 2500 \AA)$ is deposited using sputtering as the seed layer for electroplating (Fig. 3f). Subsequently, a $1 \mu \mathrm{m}$-thick Au layer is defined by optical photolithography and selectively electroplated for the anchor areas and the thick metal section in the center of the Au moving membranes (Fig. 3g). Finally, the seed layer and the sacrificial layer are removed using wet etching, and the modulator is released using critical point drying (Fig. 3h).

Figure 4a shows the schematic diagram and the scanning electron microscope (SEM) image of a fabricated terahertz modulator prototype. The meta-molecule elements are electrically connected such that the entire meta-surface structure can be switched between the modulation 'OFF' state and modulation 'ON' state, by controlling the voltage difference between the meta-surface and the underlying substrate, $V_{\text {Switch }}$. To simplify the terahertz modulator characterization, 
a

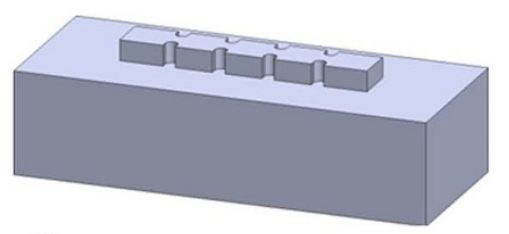

C

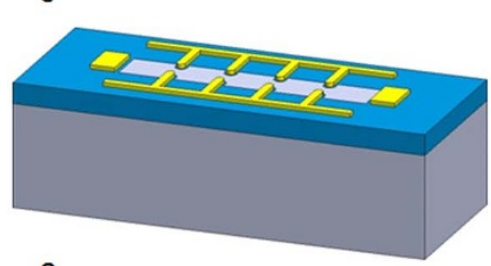

e

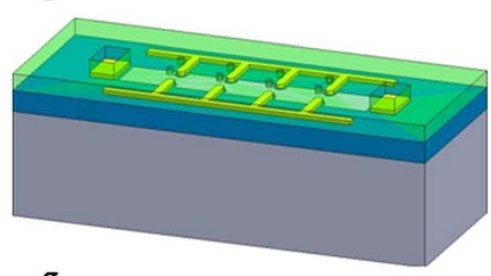

g

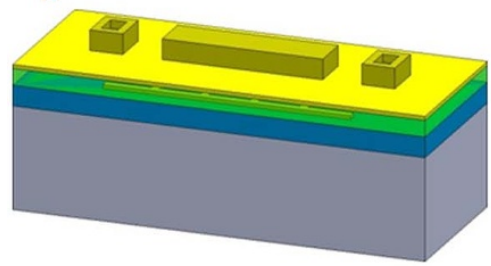

b

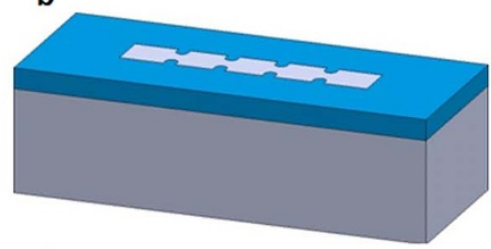

d

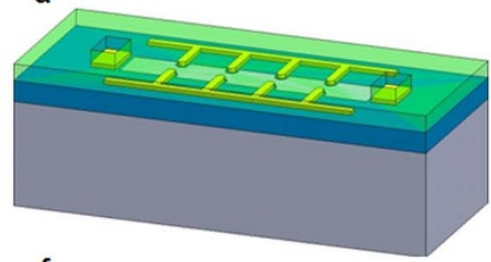

f

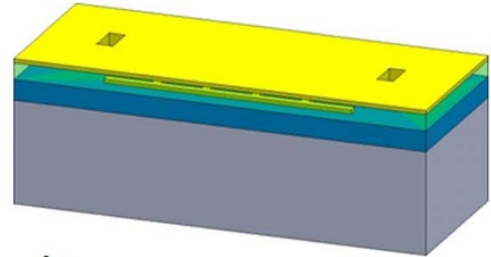

h

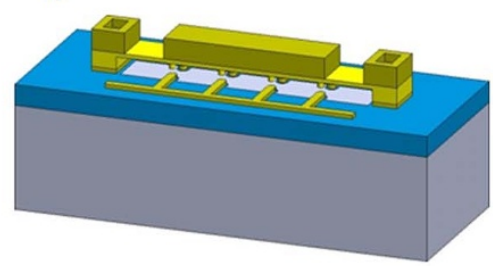

$\square$ Si substrate $\square \mathrm{SiO}_{2} \quad \square$ Sputtered Au $\square$ PMMA/PMGI $\square$ Electroplated Au

Figure 3 Flow chart of the fabrication of the meta-surface with switchable scattering parameters. (a) Defining the $\mathrm{SiO}_{2}$ isolation layer. (b) Fabricating the $\mathrm{SiO}_{2}$ layer. (c) Fabricating the horizontally-oriented Au patches and bias lines. (d) Depositing the sacrificial layer. (e) Fabricating the contact dimples. (f) Depositing the seed layer of the moving membranes. (g) Fabricating the moving membranes. (h) Removing the sacrificial layer and releasing the moving membrane.

a square metallic aperture of $\sim 1 \mathrm{~mm} \times 1 \mathrm{~mm}$ is fabricated around the meta-surface region to ensure that the transmitted terahertz power through the structure has fully interacted with the device active area. The metallic aperture also serves as the contact pad to the high-resistivity silicon substrate.

The performance of the fabricated terahertz modulator is characterized in a time-domain terahertz spectroscopy setup. By measuring the transmitted electric field of a horizontally-polarized terahertz pulse through the implemented terahertz modulator (Fig. 4b), the spectrum of the transmitted power during the modulation OFF mode $\left(V_{\text {Switch }}=0 \mathrm{~V}\right)$ and modulation ON mode $\left(V_{\text {Switch }}=30 \mathrm{~V}\right)$ is calculated, indicating more than $70 \%$ modulation depth over the $1.5 \mathrm{THz}$ frequency band (Fig. 4c). The observed spectral dips are the result of the apertures used for focusing terahertz pulses onto the modulator active area. By using larger modulator active areas larger aperture areas can be utilized, shifting the observed spectral dips to lower frequencies or completely removing them from the spectrum. By comparing the power transmission spectrum of the terahertz pulses incident on the modulator prototype with the power transmission spectrum of the terahertz pulses incident on the Si substrate through the same aperture, the signal attenuation of the modulator prototype is calculated, indicating a signal attenuation of less than $3.3 \mathrm{~dB}$ for the $1.5 \mathrm{THz}$ frequency band. As mentioned before, $3 \mathrm{~dB}$ portion of the measured signal loss is associated with Fresnel reflections at $\mathrm{Si}$-air interfaces and can be minimized by thinning the $\mathrm{Si}$ substrate under the device active area.

The dynamic characteristics of the fabricated terahertz modulator is analyzed by measuring the electric field of the transmitted tera- hertz pulses through the modulator while alternating the applied voltage between $0 \mathrm{~V}$ and $30 \mathrm{~V}$. Figure $4 \mathrm{~d}$ shows the calculated terahertz modulation depth as a function of the modulation speed, indicating modulation speeds exceeding $20 \mathrm{KHz}$. No degradation of the modulation performance is observed during the experimental characterization of the fabricated modulator prototype under various modulation speeds in the $1-20 \mathrm{KHz}$ and after accumulating more than 50 billion modulation cycles without failure.

It should be noted that the achieved modulation depth from the fabricated modulator prototype is $\sim 20 \%$ lower than the theoretically estimated modulation depth of more than $90 \%$ in the $1.5 \mathrm{THz}$ frequency range. We attribute this mainly to fabrication misalignments, which result in a non-uniform spacing between the $\mathrm{Au}$ membrane and $\mathrm{Au}$ patches of meta-molecule elements. Fabrication misalignments prevent the required contact between the $\mathrm{Au}$ membrane and $\mathrm{Au}$ patches for a portion of the meta-molecules during the modulation 'ON' mode. This hypothesis is supported by the observed increase in the measured modulation depths at higher frequencies, reaching $85 \%$ modulation depth in the 1$1.5 \mathrm{THz}$ frequency range. In spite of the capacity of the presented modulator to offer higher modulation depths through optimized fabrication processes, the achieved modulation depth is the highest reported among previously demonstrated terahertz intensity modulators, in general ${ }^{30}$, and one order-of-magnitude higher than the demonstrated broadband terahertz modulators with similar modulation voltages and modulation speeds, specifically ${ }^{31}$. The unprecedented modulation depth and modulation bandwidth of the implemented modulator is made possible by the significant change 


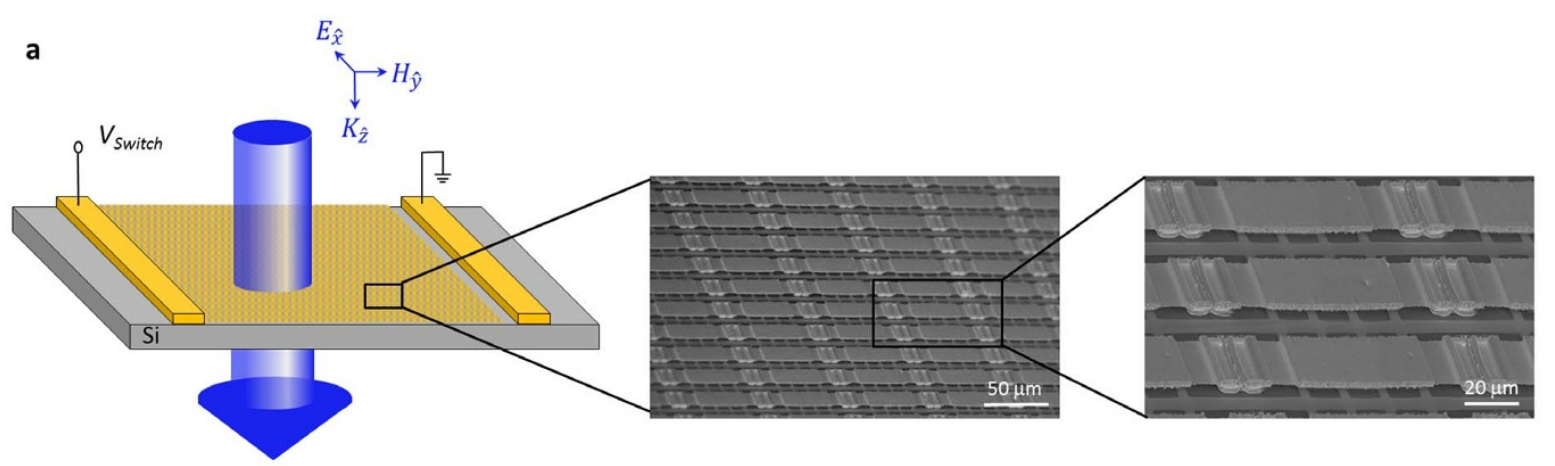

b

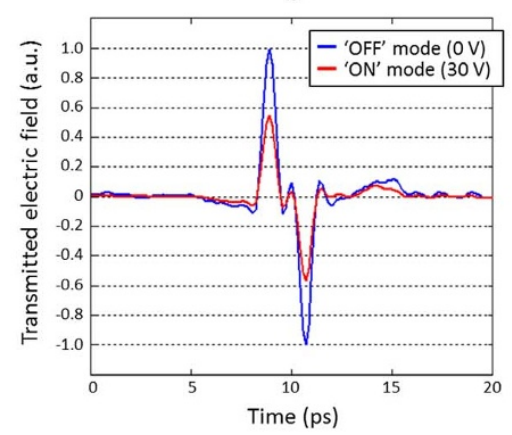

C

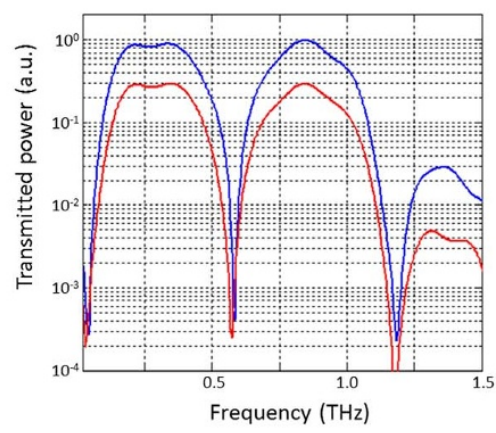

d

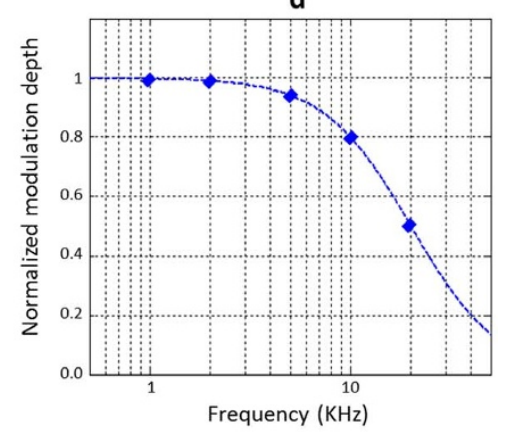

Figure $4 \mid$ Performance of the proof-of-concept terahertz modulator based on the designed meta-surface with switchable scattering parameters.

(a) The schematic diagram and the SEM image of the fabricated terahertz modulator prototype. (b) Electric field of a transmitted terahertz pulse through the modulator prototype at an applied voltage of $0 \mathrm{~V}$ (modulation OFF mode) and $30 \mathrm{~V}$ (modulation ON mode). (c) The power transmission spectrum of the terahertz pulse incident on the modulator prototype, indicating a modulation depth of more than 70\% between the modulation OFF mode and modulation ON mode. The observed spectral dips are the result of the apertures used for focusing terahertz pulses onto the device under test.

(d) The modulation depth of the fabricated prototype as a function of the modulation speed, indicating modulation speeds exceeding $20 \mathrm{KHz}$.

in the scattering parameters of the designed meta-surface over a broad frequency band.

\section{Discussion}

Existing modulation schemes in the visible and infrared regime based on carrier injection/depletion in solid-state devices ${ }^{32,33}$, MachZehnder interferometers ${ }^{34}$, Fabry-Perot filters ${ }^{35}$, liquid crystals $^{36}$, magneto-optic effects ${ }^{37}$, deformable mirrors ${ }^{38}$, and beam deflectors $^{39,40}$, have difficulty in offering high-performance modulation specifications at terahertz frequencies due to the lack of materials with the desired properties at terahertz frequencies and the practical challenges in scaling device dimensions to operate efficiently in the terahertz regime. As a result, they offer a tradeoff between the modulation depth, modulation bandwidth, modulation speed, modulation voltage, and signal attenuation when adapted for operation at terahertz frequencies ${ }^{4-46}$. On the other hand, reconfigurable metamaterials offer a very promising platform for manipulating terahertz waves $^{47-52}$ since their spectral response can be engineered by their geometry, rather than being limited by the characteristics of natural materials at terahertz frequencies. However, the operation bandwidth of the demonstrated metamaterial-based terahertz modulators has been limited by the resonant nature of the employed device configurations. There is a good reason for using resonant structures in previously demonstrated reconfigurable metamaterials: The response of a resonant structure varies considerably at its resonance frequency as a result of insignificant changes in the properties of the phase-change materials, semiconductors, graphene, superconductors, and electromechanical structures embedded in the metamaterials structure when activated by optical, electrical, magnetic, and thermal stimuli.

In contrary to previously demonstrated reconfigurable metamaterials, our presented reconfigurable meta-surface is unique in offer- ing extreme switching in the scattering parameters without use of any resonant enhancement within the device operation frequency range and, therefore, enables broadband operation. Moreover, the demonstrated broadband switching of the scattering parameters is achieved through a fully integrated, electrically controlled, room temperature platform, making it suitable for real-world and practical wave manipulation applications. The principles of the presented meta-surface with switchable scattering parameters are universal and can be extended to optical and infrared frequency ranges by further structural miniaturization. Additionally, the switching speed of the proofof-concept reconfigurable meta-surface, which has been limited by the restoring force of the designed $\mathrm{Au}$ membranes, can be further increased to gigahertz-range switching speeds by structural miniaturization or use of higher spring constant membranes, like graphene ${ }^{53}$.

1. Simpson, R. E. et al. Interfacial phase-change memory. Nat. Nanotech. 6, 501-505 (2011).

2. Driscoll, T. et al. Memory metamaterials. Science 325, 1518-1521 (2009).

3. Seo, M. et al. Active terahertz nanoantennas based on $\mathrm{VO}_{2}$ phase transition. Nano Lett. 10, 2064-2068 (2010).

4. Kampfrath, T. et al. Coherent terahertz control of antiferromagnetic spin waves. Nat. Photon. 5, 31-34 (2011).

5. Chen, H. T. et al. Active terahertz metamaterials devices. Nature 444, 597-600 (2006).

6. Zhang, S. et al. Photoinduced handedness switching in terahertz chiral metamolecules. Nat. Commun. 3, 942 (2012).

7. Chen, H. T. et al. A metamaterial solid-state terahertz phase modulator. Nat. photon. 3, 148-151 (2009).

8. Yan, H. G. et al. Tunable infrared plasmonic devices using graphene/insulator stacks. Nat. Nanotech. 7, 330-334 (2012).

9. Ju, L. et al. Graphene plasmonics for tunable terahertz metamaterials. Nat. Nanotech. 6, 630-634 (2011).

10. Lee, S. H. et al. Switching terahertz waves with gate-controlled active graphene metamaterials. Nat. Mater. 11, 936-941 (2012). 
11. Gomory, F. et al. A. Experimental realization of a magnetic cloak. Science 335, 1466-1468 (2012).

12. Ricci, M., Orloff, N. \& Anlage, S. M. Superconducting metamaterials. Appl. Phys. Lett. 87, 034102 (2005)

13. Magnus, F. et al. A d.c. magnetic metamaterials. Nat. Mater. 7, 295-297 (2008).

14. Pryce, I. M., Aydin, K., Kelaita, Y. A., Briggs, R. M. \& Atwater, H. A. Highly strained compliant optical metamaterials with large frequency tunability. Nano Lett. 10, 4222-4227 (2010).

15. Berry, C. W., Moore, J. \& Jarrahi, M. Design of reconfigurable metallic slits for terahertz beam modulation. Opt. Express 19, 1236-1245 (2011).

16. Liu, A. Q., Zhu, W. M., Tsai, D. P. \& Zheludev, N. I. Micromachined tunable metamaterials: a review. J. Opt. 14, 114009 (2012).

17. Zheludev, N. I. \& Kivshar, Y. S. From metamaterials to metadevices. Nat. Mater. 11, 917-924 (2012).

18. Ou, J.-Y., Plum, E., Zhang, J. \& Zheludev, N. I. An electromechanically reconfigurable plasmonic metamaterial operating in the near-infrared. Nat. Nano. 8, 252-255 (2013).

19. Kan, T. et al. Spiral metamaterial for active tuning of optical activity. Appl. Phys. Lett. 102, 221906 (2013).

20. Debogovic, T. \& Perruisseau-Carrier, J. MEMS-Reconfigurable Metamaterials and Antenna Applications. Int. J. Antennas Prop. 2014, 138138 (2014).

21. Zhu, W. M. et al. Microelectromechanical Maltese-cross metamaterial with tunable terahertz anisotropy. Nat. Commun. 3, doi:10.1038/ncomms2285 (2012).

22. London, I. F. Superfluids: Macroscopic Theory of Superconductivity (Dover, 1961)

23. Takagi, H. \& Hwang, H. Y. An emergent change of phase for electronics. Science 327, 1601-1602 (2010)

24. Morin, F. J. Oxides which show a metal-to-insulator transition at the Neel temperature. Phys. Rev. Lett. 3, 34 (1959).

25. Stefanovich, G., Pergament, A. \& Stefanovich, D. Electrical switching and Mott transition in $\mathrm{VO}_{2}$. J. Phys. Condens. Matter. 12, 8837-8845 (2000).

26. Cavalleri, A. et al. Femtosecond structural dynamics in $\mathrm{VO}_{2}$ during an ultrafast solid-solid phase transition. Phys. Rev. Lett. 87, 3237401 (2001).

27. Muldavin, J. B. \& Rebeiz, G. M. All-metal high-isolation series and series/shunt MEMS switches. IEEE Microwave Wireless Comp. Lett. 11, 373-375 (2001).

28. O. Luukkonen, O. et al. Simple and accurate analytical model of planar grids and high-impedance surfaces comprising metal strips or patches. IEEE Trans. Antennas Prop. 56, 1624-1632 (2008)

29. Maboudian, R. \& Howe, R. T. Critical Review: Adhesion in surface micromechanical structures. J. Vac. Sci. Technol. B. 15, 1-20 (1997).

30. Rahm, M., Li, J. S. \& Padilla, W. J. THz Wave Modulators: A brief review on different modulation techniques. J. Infrared Millimeter and Terahertz Waves 34, 1-27 (2012).

31. Sensale-Rodriguez, B. et al. Broadband graphene terahertz modulators enabled by intraband transitions. Nat. Commun. 3, 780 (2012).

32. Liu, A. et al. A high-speed silicon optical modulator based on a metal-oxidesemiconductor capacitor. Nature 427, 615-618 (2004).

33. Liu, M. et al. A graphene-based broadband optical modulator. Nature 474, 64-67 (2011).

34. Jarrahi, M., Lee, T. H. \& Miller, D. A. B. Wideband, low driving voltage traveling wave Mach-Zehnder modulator for RF photonics. Photon. Technol. Lett. 20, 517 (2008).

35. Lammel, G., Schweizer, S., Schiesser, S. \& Renaud, P. Tunable optical filter of porous silicon as key component for a MEMS spectrometer. J. Microelectromechanic. Sys. 11, 815-828 (2002).

36. Wiener, A. M., Leaird, D. E., Patel, J. S. \& Wullert, J. R. Programmable shaping of femtosecond optical pulses by use of 128 -element liquid crystal phase modulator. IEEE J. Quantum Electron. 28, 908-920 (1992).

37. Ross, W. E., Psaltis, D. \& Anderson, R. H. Two-dimensional magneto-optic spatial light modulator for signal processing. Opt. Eng. 22, 485-490 (1983).

38. Solgaard, O., Sandejas, F. S. A. \& Bloom, D. M. Deformable grating optical modulator. Opt. Lett. 17, 688-690 (1992).

39. Jarrahi, M., Pease, R. F. W., Miller, D. A. B. \& Lee, T. H. Optical switching based on high-speed phased-array optical beam steering. Appl. Phys. Lett. 92, 014106 (2008).

40. Jarrahi, M., Pease, R. F. W., Miller, D. A. B. \& Lee, T. H. High-speed optical beamsteering based on phase-arrayed waveguides. J. Vacuum Sci. Technol. B 26, 2124 (2008).
41. Drysdale, T. et al. Transmittance of a tunable filter at terahertz frequencies. Appl. Phys. Lett. 85, 5173-5175 (2004).

42. Wu, H.-Y., Hsieh, C.-F., Tang, T.-T., Pan, R.-P. \& Pan, C.-L. Electrically tunable room-temperature 2 liquid crystal terahertz phase shifter. Photon. Technol. Lett. 18, 1488-1490 (2006).

43. Cooke, D. G. \& Jepsen, P. U. Optical modulation of terahertz pulses in a parallel plate waveguide. Opt. Express 16, 15123-15129 (2008).

44. Kuzel, P., Kadlec, F., Petzelt, J., Schubert, J. \& Panaitov, G. Highly tunable SrTiO3/ DyScO3 heterostructures for applications in the terahertz range. Appl. Phys. Lett. 91, 232911 (2007)

45. Kersting, R., Strasser, G. \& Unterrainer, K. Terahertz phase modulator. Electron. Lett. 36, 1156-1158 (2000)

46. Kleine-Ostmann, T., Dawson, P., Pierz, K., Hein, G. \& Koch, M. Roomtemperature operation of an electrically driven terahertz modulator. Appl. Phys. Lett. 84, 3555-3557 (2004)

47. Tao, H., Padilla, W. J., Zhang, X. \& Averitt, R. D. Recent progress in electromagnetic metamaterial devices for terahertz applications. J. Select. Topic. Quantum Electron. 17, 92-101 (2011)

48. Chen, H. T. et al. Ultrafast optical switching of terahertz metamaterials fabricated on ErAs/GaAs nanoisland superlattices. Opt. Lett. 32, 1620-1622 (2007).

49. Chan, W. L. et al. A spatial light modulator for terahertz beams. Appl. Phys. Lett. 94, 253111 (2009).

50. Yen, T. J. et al. Terahertz Magnetic Response from Artificial Materials. Science 303, 1494-1496 (2004)

51. Padilla, W. J., Taylor, A. J., Highstrete, C., Lee, M. \& Averitt, R. D. Dynamical electric and magnetic metamaterial response at terahertz frequencies. Phys. Rev. Lett. 96, 107401 (2006).

52. Tao, H. et al. Reconfigurable terahertz metamaterials. Phys. Rev. Lett. 103, 147401 (2009).

53. Frank, I. W., Tanenbaum, D. M., van der Zande, A. M. \& McEuen, P. L. Mechanical properties of suspended graphene sheets. J. Vac. Sci. Technol. B. 25, 2558-2561 (2007).

\section{Acknowledgments}

Jarrahi's group at the University of Michigan would like to thank Prof. Anthony Grbic for useful discussions and the Lurie Nanofabrication Facility staff members for their help with device fabrication. We gratefully acknowledge the financial support from National Science Foundation Sensor and Sensing Systems division (Contract \# 1030270) and Army Research Office Young Investigator Award (Contract \# W911NF-12-1-0253).

\section{Author contributions}

M.U. fabricated the device prototypes. M.R.H. performed the electromagnetic analysis. M.U. and M.R.H. contributed equally to this work. C.W.B. performed the measurements S.L. assisted with the electromagnetic analysis. S.-H.Y. assisted with device fabrication. M.J. came up with the idea, supervised the project, and wrote the manuscript. All authors discussed the results and commented on the manuscript.

\section{Additional information}

Supplementary information accompanies this paper at http://www.nature.com/ scientificreports

Competing financial interests: The authors declare no competing financial interests.

How to cite this article: Unlu, M. et al. Switchable Scattering Meta-Surfaces for Broadband Terahertz Modulation. Sci. Rep. 4, 5708; DOI:10.1038/srep05708 (2014).

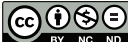

This work is licensed under a Creative Commons Attribution-NonCommercialNoDerivs 4.0 International License. The images or other third party material in this article are included in the article's Creative Commons license, unless indicated otherwise in the credit line; if the material is not included under the Creative Commons license, users will need to obtain permission from the license holder in order to reproduce the material. To view a copy of this license, visit http:// creativecommons.org/licenses/by-nc-nd/4.0/ 